\title{
A Point Mass in a Einstein Universe
}

\author{
Clement Leibovitz \\ Theoretical Physics Institute, Department of Physics, University of Alberta, \\ Edmonton, Canada
}

Received December 1, 1969

\begin{abstract}
An exact solution of Einstein's equations is presented for a perfect fluid representing a point mass in a Einstein Universe.
\end{abstract}

Unlike De Sitter's and Friedman's cosmological solutions for which a generalization with a point mass is known to exist $[1,2]$, such corresponding generalizations was not yet found for the Einstein Solution. This gap is here filled.

Let us consider the line element given by

$$
\begin{aligned}
d s^{2}=-\left(1-\frac{2 m}{r}\right)^{-1} & {\left[1-a(r-m)^{2}\right]^{-1} d r^{2} } \\
& -r^{2}\left(d \theta^{2}+\sin ^{2} \theta d \phi^{2}\right)+\left(1-\frac{2 m}{r}\right) d t^{2} .
\end{aligned}
$$

The calculated components of the energy-momentum tensors are

$$
\begin{gathered}
-8 \pi\left(T_{1}^{1}=T_{2}^{2}=T_{3}^{3}\right)=\frac{-a(r-m)^{2}}{r^{2}}, \\
8 \pi \varrho=\frac{a}{r^{2}}(3 r-5 m)(r-m) .
\end{gathered}
$$

It represents therefore an ideal fluid. For $a=0$ the element reduces to that of the Schwarzschild's exterior solution. For $m=0$ we have the Einstein universe.

The correct signature is obtained for

$$
2 m<r \quad \text { and } \quad(r-m)^{2}<\frac{1}{a} .
$$

The two inequalities are compatible for $m^{2} a<1$. Provided that this last inequality is satisfied it is possible to find a range of values for $r$ so that (4) holds. 
The density and the pressure are of opposite sign. However, whenever the inequalities (4) are satisfied we have in absolute value $|\varrho|>|p|$. As $r$ approach the value $2 m$, the absolute values of $\varrho$ and $p$ become equal.

Note added in proof. This solution cannot be reduced to the static particular case of the one given in reference [2].

\section{References}

1. Tolman, R. C.: Relativity thermodynamics and cosmology, p. 245. Equation 96.3 gives the line element of Schwarzschild-De Sitter solution. Oxford 1934.

2. McVittie, G. C.: Monthly Notices Roy. Astron. Soc. 93, 325 (1933). It gives the line element for a point mass in a Friedman universe.

C. Leibovitz

Theoretical Institute

Department of Physics

University of Alberta

Edmonton, Canada 